\title{
A validated Geant4 model of a whole-body PET scanner with four-layer DOI detectors
}

To cite this article before publication: Abdella M Ahmed et al 2020 Phys. Med. Biol. in press https://doi.org/10.1088/1361-6560/abaa24

\section{Manuscript version: Accepted Manuscript}

Accepted Manuscript is "the version of the article accepted for publication including all changes made as a result of the peer review process, and which may also include the addition to the article by IOP Publishing of a header, an article ID, a cover sheet and/or an 'Accepted

Manuscript' watermark, but excluding any other editing, typesetting or other changes made by IOP Publishing and/or its licensors"

This Accepted Manuscript is (c) 2020 Institute of Physics and Engineering in Medicine.

During the embargo period (the 12 month period from the publication of the Version of Record of this article), the Accepted Manuscript is fully protected by copyright and cannot be reused or reposted elsewhere.

As the Version of Record of this article is going to be / has been published on a subscription basis, this Accepted Manuscript is available for reuse under a CC BY-NC-ND 3.0 licence after the 12 month embargo period.

After the embargo period, everyone is permitted to use copy and redistribute this article for non-commercial purposes only, provided that they adhere to all the terms of the licence https://creativecommons.org/licences/by-nc-nd/3.0

Although reasonable endeavours have been taken to obtain all necessary permissions from third parties to include their copyrighted content within this article, their full citation and copyright line may not be present in this Accepted Manuscript version. Before using any content from this article, please refer to the Version of Record on IOPscience once published for full citation and copyright details, as permissions will likely be required. All third party content is fully copyright protected, unless specifically stated otherwise in the figure caption in the Version of Record.

View the article online for updates and enhancements. 


\section{A validated Geant4 model of a whole-body PET} z scanner with four-layer DOI detectors

Abdella M. Ahmed ${ }^{1,2}$, Andrew Chacon ${ }^{1,2}$, Harley Rutherford ${ }^{1,2}$, Go Akamatsu ${ }^{3}$, Akram Mohammadi ${ }^{3}$, Fumihiko Nishikido ${ }^{3}$, Hideaki Tashima $^{3}$, Eiji Yoshida ${ }^{3}$, Taiga Yamaya ${ }^{3}$, Daniel R. Franklin $^{4}$, Anatoly Rosenfeld ${ }^{2,5}$, Susanna Guatelli ${ }^{2,5}$, Mitra Safavi-Naeini ${ }^{1,2,6}$

1 Australian Nuclear Science and Technology Organisation(ANSTO), NSW, Australia

${ }^{2}$ Centre for Medical Radiation Physics, University of Wollongong, Wollongong NSW 2522 Australia

${ }^{3}$ National Institute of Radiological Sciences (NIRS), National Institutes for Quantum and Radiological Science and Technology, 4-9-1 Anagawa, Inage-ku, Chiba 263-8555, Japan

${ }^{4}$ School of Electrical and Data Engineering, University of Technology Sydney, Australia

${ }^{5}$ Illawarra Health and Medical Research Institute, University of Wollongong, Wollongong NSW 2522 Australia

${ }^{6}$ Brain and Mind Centre, University of Sydney, Sydney, NSW, Australia

E-mail: abdellaa@ansto.gov.au

E-mail: mitras@ansto.gov.au 
A validated Geant4 model of a whole-body PET scanner with four-layer DOI detectors 2

\begin{abstract}
The purpose of this work is to develop a validated Geant4 simulation model of a whole-body prototype PET scanner constructed from the four-layer depthof-interaction detectors developed at the National Institute of Radiological Sciences, National Institutes for Quantum and Radiological Science and Technology, Japan. The simulation model emulates the behaviour of the unique depth of interaction sensing capability of the scanner without needing to directly simulate optical photon transport in the scintillator and photodetector modules. The model was validated by evaluating and comparing performance metrics from the NEMA NU 2-2012 protocol on both the simulated and physical scanner, including spatial resolution, sensitivity, scatter fraction, noise equivalent count rates and image quality. The results show that the average sensitivities of the scanner in the field-of-yiew were $5.9 \mathrm{cps} / \mathrm{kBq}$ and $6.0 \mathrm{cps} / \mathrm{kBq}$ for experiment and simulation, respectively. The average spatial resolutions measured for point sources placed at several radial offsets were $5.2 \pm 0.7 \mathrm{~mm}$ and $5.0 \pm 0.8 \mathrm{~mm}$ FWHM for experiment and simulation, respectively. The peak NECR was $22.9 \mathrm{kcps}$ at $7.4 \mathrm{kBq} / \mathrm{mL}$ for the experiment, while the NECR obtained via simulation was $23.3 \mathrm{kcps}$ at the same activity. The scatter fractions were $44 \%$ and $41.3 \%$ for the experiment and simulation, respectively. Contrast recovery estimates performed in different regions of a simulated image quality phantom matched the experimental results with an average error of $-8.7 \%$ and $+3.4 \%$ for hot and cold lesions, respectively. The results demonstrate that the developed Geant 4 model reliably reproduces the key NEMA NU 2-2012 performance metrics evaluated on the prototype PET scanner. A simplified version of the model is included as an advanced example in Geant4 version 10.5.
\end{abstract}

\title{
1. Introduction
}

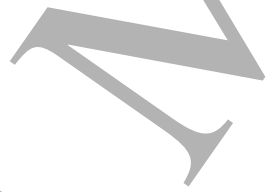

Positron emission tomography (PET) is a non-invasive nuclear medicine technique that is used for the clinical diagnosis of cancer and the study of a range of diseases and biochemical processes in living organisms. The quality of reconstructed PET images is limited by the amount of activity in the object, the duration of the scan, and the performance of the PET scanner - which, in turn, depends on its constituent components, such as the type and size of scintillator material used, the detection efficiency, geometrical arrangement of the detectors and the readout electronics. In addition, the choice of parameters for data acquisition (such as acquisition time, energy window, and coincidence timing window) and reconstruction (choice of algorithm, number of subsets, number of iterations etc.) also affect the quality of the reconstructed image. Experimental optimisation of these parameters is very expensive in terms of time, materials and labour.

Monte Carlo simulation provides a versatile and low-cost alternative to experimental optimisation of imaging parameters. High-fidelity simulations of existing physical scanners, validated for correctness against experimental measurements, enable the development of new image reconstruction algorithms, segmentation methods and optimised imaging protocols for quantitative evaluation of radiotracer uptake metrics. A simulation-based approach enables quantitative imaging experiments to be planned with advance knowledge of the achievable signal to noise ratio and other signal quality 


\section{A validated Geant4 model of a whole-body PET scanner with four-layer DOI detectors3}

metrics, without wasting time, radiotracer and sacrificed animals on potentially futile experiments $[1,2,3,4,5]$. This approach has the advantage of perfect repeatability for example, exactly the same spatio-temporal radiotracer uptake distribution can be imaged with different scanner parameters (for example, timing and energy windows).

A wide range of Monte Carlo simulation platforms have been developed for this purpose including the Geant4 Application for Tomographic Emission (GATE) [6, 7, 8], Simulation System for Emission Tomography (SimSET) [9, 10, 11], Geant4-based Architecture for Medicine-Oriented Simulations (GAMOS) [12, 13] and a PET-dedicated Monte Carlo tool based on PENELOPE (PeneloPET) [14].

The performance of clinical PET scanners is quantified using the National Electrical Manufacturers Association (NEMA) NU 2 standard, first published in 1994 and most recently updated in 2012 and 2018 [15, 16, 17]. The NEMA NU 2 standard provides a suite of protocols for the standardised measurement of spatial resolution, sensitivity, scatter fraction, count losses and random measurements, and the accuracy of attenuation and scatter correction for image quality. Many validation works for Monte Carlo models of different scanners have been conducted by following the NEMA NU 2 protocols (or the related NU 4 standard for preclinical scanners) on corresponding simulated and physical scanners and comparing the results. Lamare et al. developed a simulation model of the Philips Allegro/GEMINI PET system in GATE, and its spatial resolution, noise equivalent count rate, sensitivity and scatter fraction were compared with the measured results [8]. Gonias et al. compared scatter fractions obtained using the Siemens Biograph mCT PET scanner and its corresponding GATE model, obtaining good agreement while observing that decreasing the dead-time of the simulation improved the accuracy of the simulation's scatter fraction estimate [18]. Poon et al. performed NEMA validation of the scatter fraction, count rates and spatial resolution obtained using a SimSET model of the same Siemens Biograph $\mathrm{mCT}$ scanner and compared these results with experimental data [11]. Ghabrial et al. validated a GATE simulation model, also of the Siemens Biograph mCT PET/CT scanner, using the NEMA NU 2 protocols, finding that the spatial resolution and sensitivity obtained from the simulation tended to underestimate experimental values, while scatter fraction was slightly overestimated [19].

The purpose of this work is to develop and validate a Geant4 simulation model of the world's first whole-body/prototype PET scanner constructed from 4-layer depth-ofinteraction (DOI) detectors developed at the National Institute of Radiological Sciences (NIRS), National Institutes for Quantum and Radiological Science and Technology, Japan. This scanner is specifically designed for in-beam clinical quality assurance in heayy ion therapy, in which the positron-emitting radioactive fragments produced during therapy are imaged in order to provide range verification information. The developed model can be used by researchers to help plan and optimise imaging experiments to be performed with the heavy ion beamlines at NIRS; it will also be a valuable tool to assist in the design of newer versions or configurations of the scanner.

The use of 4-layer DOI detectors allows the prototype to achieve uniformly high spatial resolution throughout the entire field of view (FOV), with excellent contrast 

recovery in axially-offset regions with limited radiotracer uptake. Utilisation of DOI information avoids the broadening of lines of response in the radial dimension which occurs near the periphery of the scanner due to parallax error. This results in improved spatial resolution in the radial direction in the reconstructed image compared to the non-DOI case, at the cost of a substantially larger system matrix (by a factor of $\mathrm{N}^{2}$ for $N$-layer DOI). The experimental performance of the 4-layer DOI detectors have been extensively evaluated by Tsuda et al. and Hirano et al. [20, 21]; the complete scanner is described and analytically modelled by Tashima et al. and experimentally characterised using the NEMA NU 2 protocols by Akamatsu et al. [22, 23].

The Geant4 Monte Carlo simulation framework was selected as the development platform due to the large body of existing particle therapy simulation code available for Geant4, and the ease with which this code may be integrated with the simulated PET scanner for complete simulation and modelling of PET quality assurance. Additionally, the model can be easily modified to incorporate other unconventional geometries (such as the Single-Ring OpenPET [22]) which are optimised for particle therapy QA; simulation of non-right-cylindrical geometries is not straightforward in GATE.

Development of a validated model for this particular scanner will be of considerable value and utility in a number of different projects. The physical scanner is used for a wide range of research, including inverse dose estimation methods [24, 25], evaluation of Geant4 hadronic ion inelastic physics models for range verificaion in particle therapy [26] and experimental evaluation of the use of positron-emitting ion beams for particle therapy $[27,28]$. Furthermore, an oblique-ring version of this scanner will be used for clinical quality assurance work at HIMAC; the proposed Monte Carlo simulation model will be useful for developing and/testing the clinical quality assurance protocols [29]. The developed model directly reflects the physical structure of the scanner, while utilising an analytic model of the light-sharing scheme used by the unique 4-layer DOI-sensing system employed in the scanner; this avoids the need to simulate optical photon transport, thereby greatly improving the speed of the simulation. Simulations were conducted according to the NEMA NU 2-2012 protocolł, and spatial resolution, sensitivity, scatter fraction (SF), noise equivalent count rates (NECR) and image quality were evaluated. The results were validated against corresponding experimental measurements obtained with the prototype PET scanner [16, 23].

A simplified version of the simulation model is now included as an advanced example in Geant4 version 10.5.

\section{Materials and Methods}

All source code developed for this project is available via the following URL: https://bitbucket.org/msafavi/whole-body-doi-pet-simulation-model.

The 2018 update of the NEMA NU 2 protocol is equivalent to the 2012 version from the perspective of the parameters evaluated in this study. 
A validated Geant4 model of a whole-body PET scanner with four-layer DOI detectors5

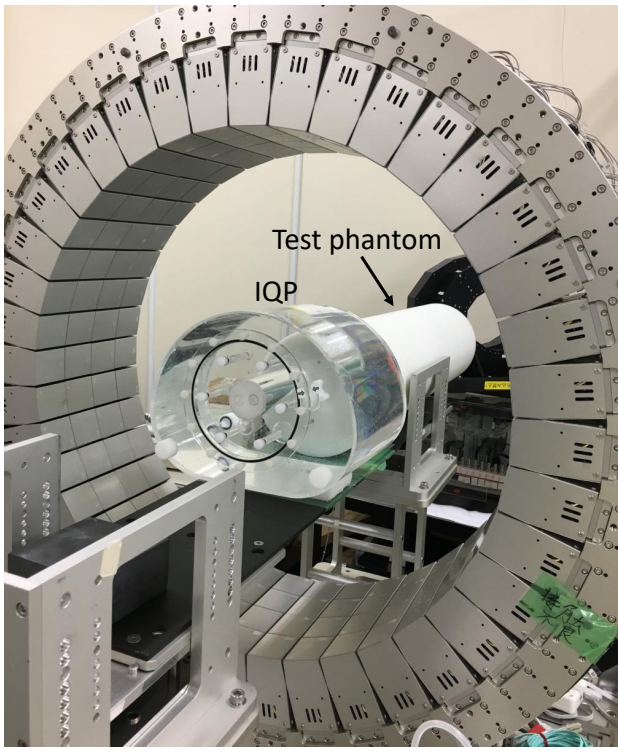

(a) Prototype scanner

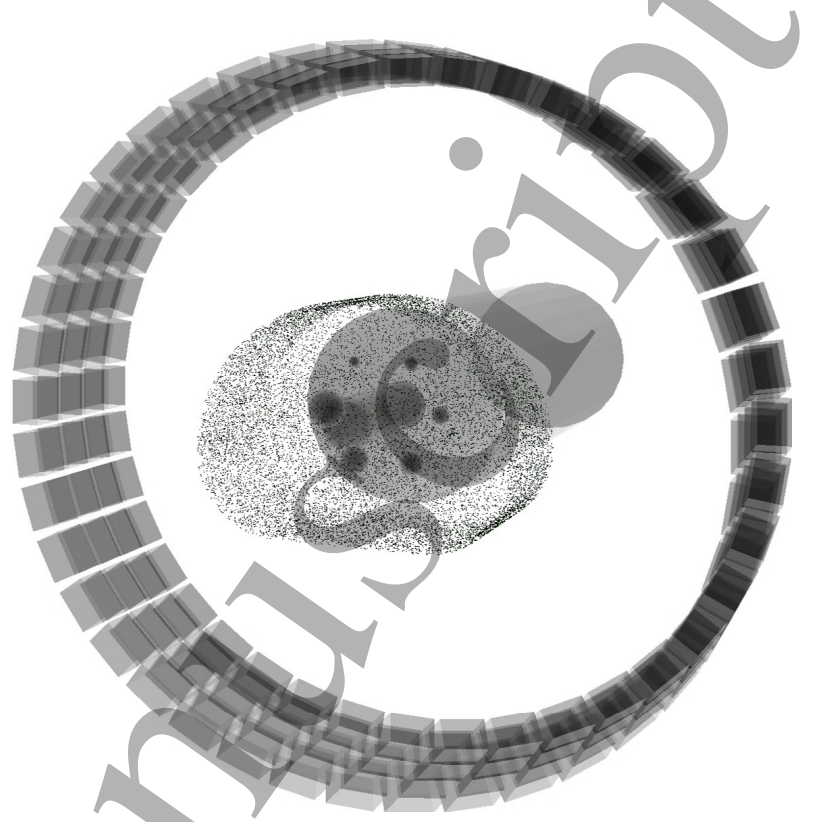

(b) Simulation

Figure 1: The whole-body DOI-PET prototype and equivalent simulation with image quality phantom (IQP) and test phantoms.

\subsection{Whole-body DOI-PET design}

The whole-body DOI-PET prototype being modelled in this work consists of four rings, each composed of 40 detector modules with DOI capability [23]. The physical PET scanner and its corresponding simulation model are shown in Figure 1(a) and Figure 1(b), respectively, and the scanner specifications are summarised in Table 1. The scanner has an internal diameter of $660 \mathrm{~mm}$ with an axial FOV of $215 \mathrm{~mm}$. Each detector module consists of a $16 \times 16 \times 4$ array of Zr-doped gadolinium oxyorthosilicate (GSOZ) scintillation crystals with dimensions of $2.8 \times 2.8 \times 7.5 \mathrm{~mm}^{3}$, with the outermost layer of the array optically coupled to a 64 -channel $(8 \times 8)$ flat panel position-sensitive photomultiplier tube (PS-PMT). Depth of interaction is determined using a light-sharing method in which the optical photon distributions arriving at the PS-PMT are modified via the insertion of radial/reflectors in the crystal array with different patterns for each layer (see Figure 4) [21]. This results in the radial component of the 3D point of interaction being encoded in the $2 \mathrm{D}$ optical photon distribution histogram as the absence or presence of a translation in the axial and/or tangential direction.

\subsection{Simulation Model}

A schematic illustration of the PET scanner (along with the NEMA NU 2 image quality phantom) is shown in Figure 2. The scanner was simulated using Geant4 toolkit version 10.5.p01; the standard physics option 3 model (G4EmStandardPhysics_option3) was used for electromagnetic interactions $[30,31]$. The simulated scanner has the same 


\section{A validated Geant4 model of a whole-body PET scanner with four-layer DOI detectors6}

Table 1: Parameters of the PET scanner

\begin{tabular}{ll}
\hline Parameter & Value \\
\hline Scintillation material & GSOZ (phys) / GSO (sim) \\
Photodetector & 64 -ch $(8 \times 8)$ flat panel PS-PMT \\
Size of crystal & $2.8 \times 2.8 \times 7.5 \mathrm{~mm}^{3}$ \\
Crystals per detector & $16 \times 16 \times 4$ \\
Ring diameter & $660 \mathrm{~mm}$ \\
Axial field-of-view & $215 \mathrm{~mm}$ \\
Number of rings & 4 \\
Number of detectors per ring & 40 \\
Time resolution & $4.4 \mathrm{~ns}$ \\
\hline
\end{tabular}

geometry as the physical scanner (Table 1). The scintillation material used in the simulation is GSO rather than GSOZ; this is because the zirconium dopant used in the prototype PET scanner is only present in trace quantities to increase the light output (by around 20\% compared with that of undoped GSO), and has an insignificant effect on the other physical properties of the scintillator [32]. The crystal block is placed inside an aluminium light shield with a thickness of $0.3 \mathrm{~mm}$. A paralysable dead-time of $256 \mathrm{~ns}$ is applied on each block. The output of the simulation is stored as single list-mode data; coincidences are detected via post-simulation analysis. The coincidence timing window is set to $10 \mathrm{~ns}$, while the energy window is $400 \mathrm{keV}-600 \mathrm{keV}$.

The list-mode coincidence data records the global crystal identification number for both endpoints of each line of response.

To greatly increase the speed of the simulation, optical photon emission and transport is not simulated; instead, the true location of any energy deposition in the scintillators is logged, and the position response of the PS-PMT and Anger logic decoding scheme is modelled analytically based on this location. This approach is fully described in Section 2.2.2; it is similar to the approach normally used by GATE, although the DOI-decoding logic is different to any of the systems supported by that simulator $[7]$.

2.2.1. Detection Efficiency Following deposition of energy in a scintillation crystal, the probability that an event is detectable depends on the scintillator light yield and emission spectrum, the transfer efficiency of the scintillator-PMT system, the quantum efficiency of the PMT and the detection threshold of the pulse detector. These terms do not need to be individually determined in the simulation to provide an accurate model of the system sensitivity. Instead, an overall detection efficiency factor for the scintillator/PMT chain is estimated by performing a sensitivity measurement on the simulated scanner (based on the NEMA NU 2-2012 sensitivity scan) with an axial line source at low activity (so as to eliminate the impact of detector module dead-time) at the centre of the scanner 
A validated Geant4 model of a whole-body PET scanner with four-layer DOI detectors 7

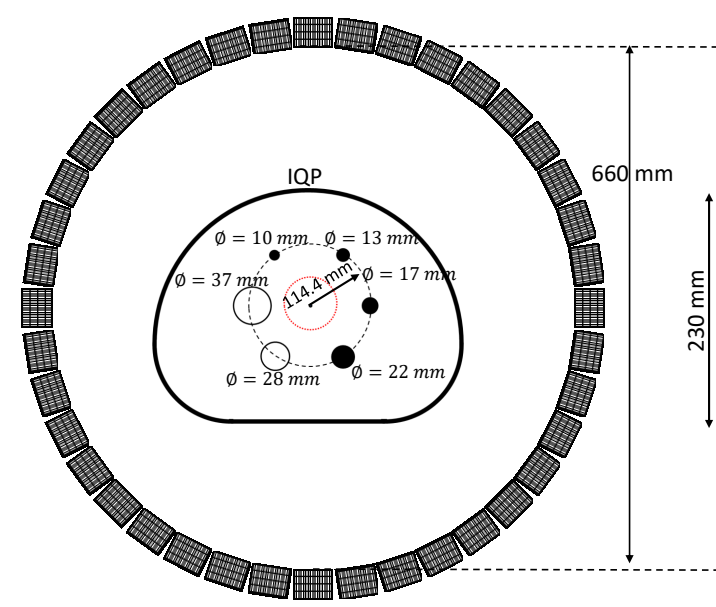

(a)

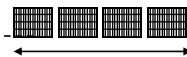

$215 \mathrm{~mm}$

IQP

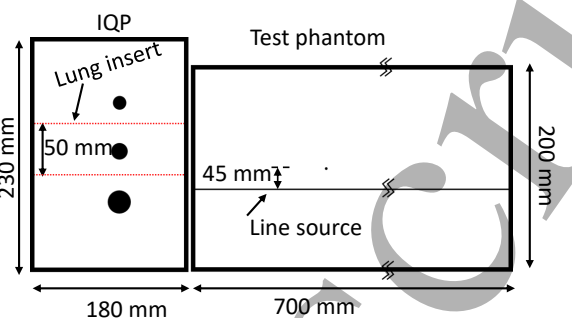

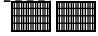

Figure 2: Schematic illustration of the PET scanner with NEMA NU2 image quality phantom (IQP). The red circle at the centre of the FOV represents the lung insert, filled black circles are "hot" lesions and those with no colour are "cold" lesions. (a) Transversal view, (b) axial view, where the test phantom is placed next to the IQP.

and an assumed detection probability of $100 \%$, then reducing the proportion of detected events until the mean detection count rate per unit source activity density matches the experimentally observed sensitivity. This detection probability value was found to be $88 \%$.

\subsubsection{Interaction Localisation and Error Modelling Full simulation of optical photon} emission and transport is not performed due to the high computational burden that this would impose. Instead, the exact amount of energy deposited and the true point of interaction within a crystal for each detected event is recorded in the simulation, and deliberately degraded according to a model of the pulse processing and DOI estimation system implemented in the actual scanner to produce a realistic error distribution.

The effective energy resolutions of individual scintillator crystals in the simulation are randomly generated at the start of the simulation and stored in a look-up table. This per-crystal parameter combines the variations in energy resolution between the energy resolution of individual scintillation crystals, optical coupling efficiencies and PMT gains of a real scanner as a single Gaussian random variable representing overall effective energy resolution. The mean value of this effective energy resolution was set to $15 \%$ with a standard deviation of $1 \%$ (such that more than $95 \%$ of crystals had an effective energy resolution between $13 \%$ and $17 \%$ ); these values were based on measurements obtained from the physical scanner.

In the physical scanner, the depth of interaction is encoded using a pattern of reflectors inserted between adjacent pairs of crystals in each layer, with the pattern of reflectors shifted by one crystal axially in the second layer, one crystal tangentially in the third layer, and then one crystal in both dimensions in the fourth layer [20]. In 


\section{A validated Geant4 model of a whole-body PET scanner with four-layer DOI detectors 8}

this way, the interacting layer can be determined by observing the shift of the optical photon distribution observed at the base of the bottom layer, which is optically coupled to a position-sensitive PMT. To simplify the electronics, the 64 anodes of the PMT (Hamamatsu H9500) are combined via an Anger logic resistive ladder network to four electrical outputs. If all energy is deposited in a single interaction, the interacting crystal may be uniquely identified by decoding the Anger logic signals via analysis of the relative pulse heights; if the energy is distributed between multiple crystals, the centroid of the resulting projections is used to assign the endpoint location.

A simplified diagram which illustrates crystal identification and DOI quantification is shown in Figure 4 for a 4-layer DOI detector with an $8 \times 8$ crystal array coupled to an ideal position-sensitive PMT with Anger-logic readout at each corner (Figure 3 (a)). The reflector pattern for each layer and the expected position response are shown in Figure 4 (a) and Figure 4 (b), respectively. The expected position response of all layers when projected in 2D histogram is shown in Figure 4 (c). The size of the pixel in which the 2D position histogram was half of the crystal pitch. Crystal identification (and hence DOI quantification) is performed via a mapping of the $2 \mathrm{D}$ position projection (Figure 5 (a)) to a look-up table (Figure $5(\mathrm{~b})$ ).

The simulation uses the following process to emulate the physical scanner's method for determining the specific crystal with which a gamma photon has interacted:

- The spatial position $P$ of the centre of mass of the exact locations of all energy depositions in a detector block is calculated;

- The individual amounts of energy deposited in each crystal are scaled by a random gain term according to the energy resolution of each crystal, and the weighted sum E calculated;

- The crystal $C_{i, j, k}$ in which $P$ is located is identified (where $i, j$ and $k$ are the indices of the crystal's position in $x, y$ and $z$ dimensions);

- A number of optical photons $N$ emitted from $C_{i, j, k}$ is calculated (but not simulated) based on $E$ and the nominal photon yield of the crystal;

- The exact location on the PMT surface to which the optical photons are mapped due to the reflector patterns is then determined according to the $16 \times 16 \times 4$ extension of Figure 4 and 5;

- An empirically-derived error is added to this 2D location (iteratively optimised to achieve the best overall match between the simulation and experimental spatial resolutions) to model the finite signal to noise ratio of the PMT and the quantisation noise resulting from digitisation of the readout signals;

- The result coordinates are quantised to a $32 \times 32$ grid (with resolution equal to half the crystal width) and mapped back to a crystal in the scintillator array - the $x$ and $y$ index are determined by dividing the quantised location on the grid by two in each dimension, while the layer is determined by mapping the quantised location with a DOI look-up table as shown in Figure 5. 
A validated Geant4 model of a whole-body PET scanner with four-layer DOI detectors 9

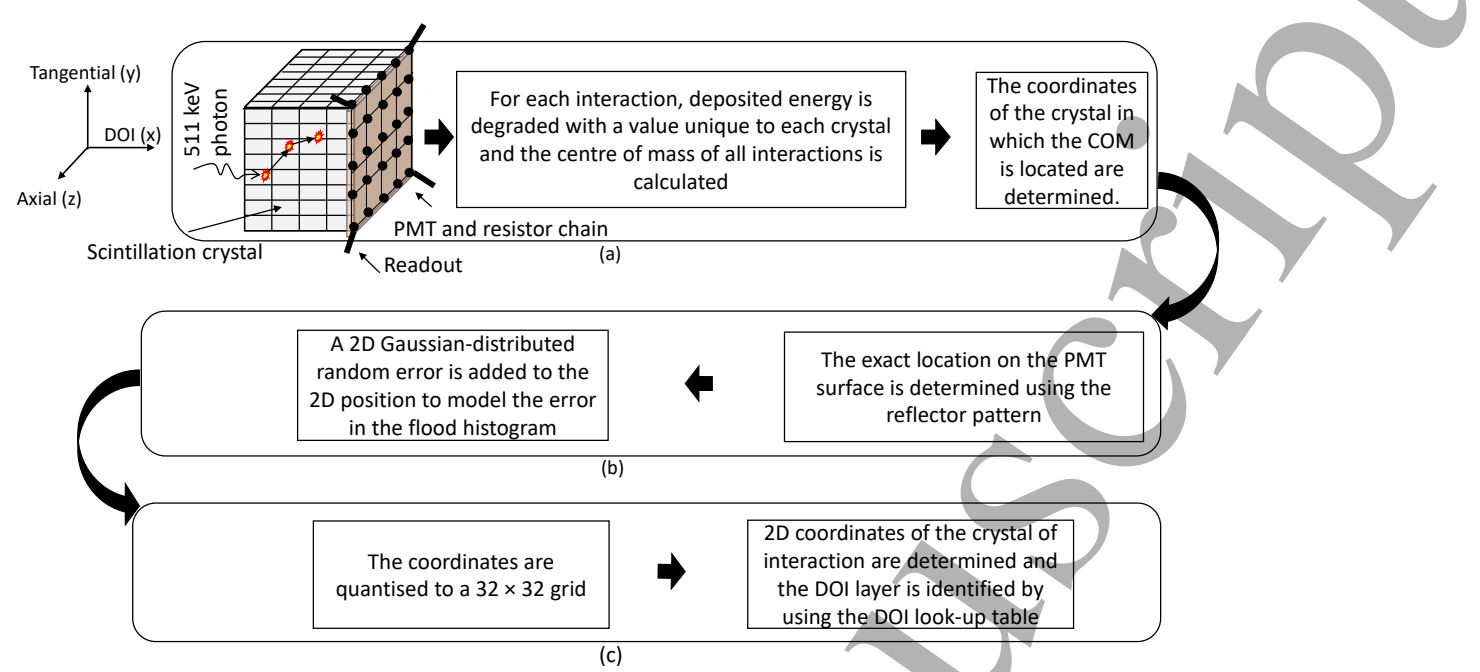

Figure 3: Flowchart illustrating the mapping of gamma photon interactions to a specific crystal within the detector block, without simulating optical photons. (a) Identification of the crystal where the centre of mass of the interactions is located, including any intercrystal scatter in the scintillator block; (b) modeling the imperfect position response of the light-sharing scintillators, PMT and Anger logic by adding a Gaussian-distributed 2D error optimised to best match the experimental scanner performance; and (c) identification of the crystal of interaction in $3 \mathrm{D}$ by using a DOI look-up table. The PMT and the resistor network are shown for illustrative purposes only.

(a)

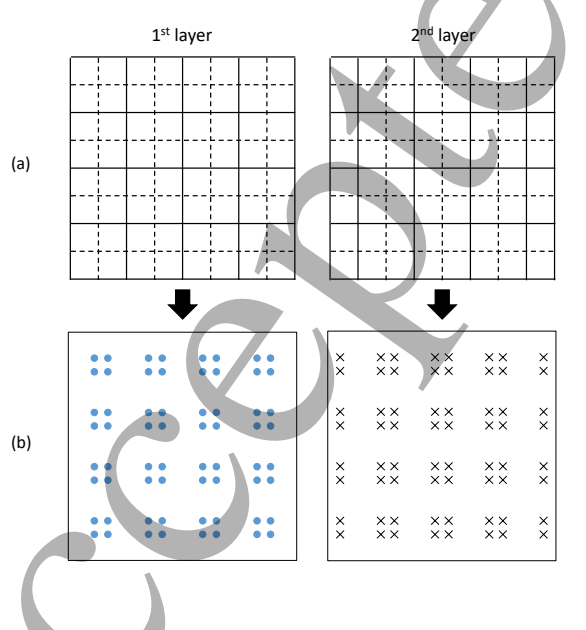

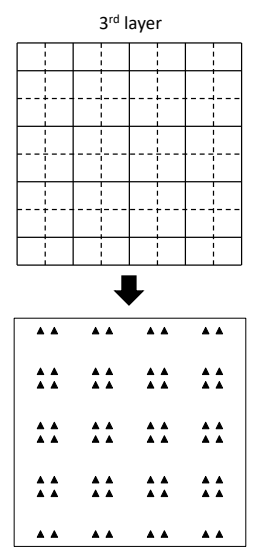

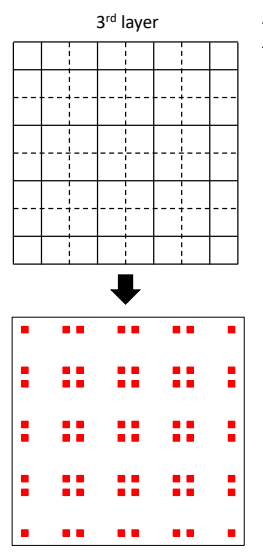

--- Air
Reflector

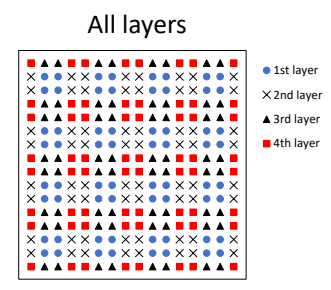

(c)

Figure 4: Illustration of position response for a four layer DOI detector with a (simplified) $8 \times 8 \times 4$ crystal array (Figure 3 (a)). (a) Reflector pattern for each layer, (b) expected $2 \mathrm{D}$ position response for each layer, and (c) the overall position response when projected in a $2 \mathrm{D}$ position histogram. The actual $16 \times 16 \times 4$ array follows an extension of the same pattern. 


\section{A validated Geant4 model of a whole-body PET scanner with four-layer DOI detectors10}

(a)

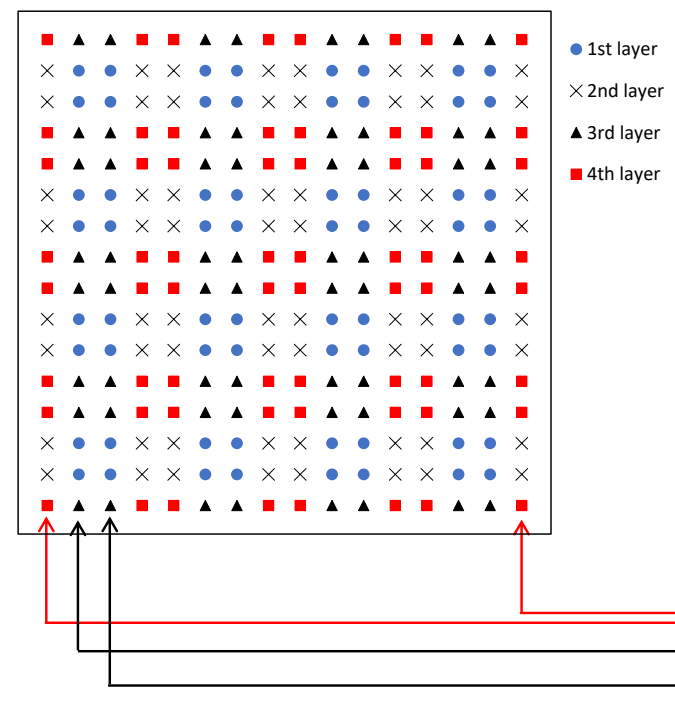

(b)

$\begin{array}{llllllllllllllll}4 & 3 & 3 & 4 & 4 & 3 & 3 & 4 & 4 & 3 & 3 & 4 & 4 & 3 & 3 & 4\end{array}$

$\begin{array}{llllllllllllllll}2 & 1 & 1 & 2 & 2 & 1 & 1 & 2 & 2 & 1 & 1 & 2 & 2 & 1 & 1 & 2\end{array}$

$\begin{array}{llllllllllllllll}2 & 1 & 1 & 2 & 2 & 1 & 1 & 2 & 2 & 1 & 1 & 2 & 2 & 1 & 1 & 2\end{array}$

$\begin{array}{llllllllllllllll}4 & 3 & 3 & 4 & 4 & 3 & 3 & 4 & 4 & 3 & 3 & 4 & 4 & 3 & 3 & 4\end{array}$

$\begin{array}{llllllllllllllll}4 & 3 & 3 & 4 & 4 & 3 & 3 & 4 & 4 & 3 & 3 & 4 & 4 & 3 & 3 & 4\end{array}$

$\begin{array}{llllllllllllllll}2 & 1 & 1 & 2 & 2 & 1 & 1 & 2 & 2 & 1 & 1 & 2 & 2 & 1 & 1 & 2\end{array}$

$\begin{array}{llllllllllllllll}2 & 1 & 1 & 2 & 2 & 1 & 1 & 2 & 2 & 1 & 1 & 2 & 2 & 1 & 1 & 2\end{array}$

$\begin{array}{llllllllllllllll}4 & 3 & 3 & 4 & 4 & 3 & 3 & 4 & 4 & 3 & 3 & 4 & 4 & 3 & 3 & 4\end{array}$

$\begin{array}{llllllllllllllll}4 & 3 & 3 & 4 & 4 & 3 & 3 & 4 & 4 & 3 & 3 & 4 & 4 & 3 & 3 & 4\end{array}$

$\begin{array}{lllllllllllllllll}2 & 1 & 1 & 2 & 2 & 1 & 1 & 2 & 2 & 1 & 1 & 2 & 2 & 1 & 1 & 2\end{array}$

$\begin{array}{llllllllllllllll}2 & 1 & 1 & 2 & 2 & 1 & 1 & 2 & 2 & 1 & 1 & 2 & 2 & 1 & 1 & 2\end{array}$

$\begin{array}{llllllllllllllll}4 & 3 & 3 & 4 & 4 & 3 & 3 & 4 & 4 & 3 & 3 & 4 & 4 & 3 & 3 & 4\end{array}$

$\begin{array}{llllllllllllllll}4 & 3 & 3 & 4 & 4 & 3 & 3 & 4 & 4 & 3 & 3 & 4 & 4 & 3 & 3 & 4\end{array}$

$\begin{array}{llllllllllllllll}2 & 1 & 1 & 2 & 2 & 1 & 1 & 2 & 2 & 1 & 1 & 2 & 2 & 1 & 1 & 2\end{array}$

$\begin{array}{llllllllllllllll}2 & 1 & 1 & 2 & 2 & 1 & 1 & 2 & 2 & 1 & 1 & 2 & 2 & 1 & 1 & 2\end{array}$

$\begin{array}{llllllllllllllll}4 & 3 & 3 & 4 & 4 & 3 & 3 & 4 & 4 & 3 & 3 & 4 & 4 & 3 & 3 & 4\end{array}$

\subsection{NEMA NU 2-2012 Performance Evaluation}

Performance of the simulated scanner was evaluated according to the NEMA NU 2-2012 protocol, and compared with experimental measurements performed on the physical scanner.

2.3.1. Sensitivity Sensitivity was evaluated by modelling a $700 \mathrm{~mm}$ long polyethylene tube (with inner and outer diameters of $1 \mathrm{~mm}$ and $3 \mathrm{~mm}$, respectively), filled with ${ }^{18} \mathrm{~F}$ solution and inserted into between one and five concentric $700 \mathrm{~mm}$ long aluminium sleeves. List-mode data was recorded for $600 \mathrm{~s}$ at two positions within the FOV: centred (with activity ranging from $2.88 \mathrm{MBq}$ to $3.85 \mathrm{MBq}$ ), and at a radial displacement of $100 \mathrm{~mm}$ (with activity ranging from $2.0 \mathrm{MBq}$ to $2.67 \mathrm{MBq}$ ). List-mode data was rebinned using the single slice rebinning algorithm, with a slice thickness of $1.5 \mathrm{~mm}$, over an axial range of $215 \mathrm{~mm}$. Sensitivity was calculated as the number of true coincidence events per unit activity. The sensitivity measurement with no attenuation was extrapolated from the data set (as per the NEMA NU 2-2012 protocol).

2.3.2. Spatial Resolution Spatial resolution was evaluated by simulating a set of pointlike cylindrical sources $(\oslash=h=1 \mathrm{~mm})$ filled with ${ }^{18} \mathrm{~F}$ solution. The point sources were placed at radial offsets of $10 \mathrm{~mm}, 100 \mathrm{~mm}$ and $200 \mathrm{~mm}$, at the centre of axial FOV (AFOV) and at $\frac{3}{8}$ of the AFOV with a total source activity of $0.61 \mathrm{MBq}$. List mode data was recorded for $600 \mathrm{~s}$. A 3D sinogram was generated with a maximum ring difference of 40 and then a 2D sinogram was generated by the Fourier rebinning algorithm (FORE). The images were reconstructed using filtered back projection (FBP), with a voxel size 
A validated Geant4 model of a whole-body PET scanner with four-layer DOI detectors 11

of $1 \times 1 \times 1 \mathrm{~mm}^{3}$. Spatial resolution was measured as the full width at half maximum (FWHM) of the point source for the radial, tangential and axial directions. The spatial resolution was also evaluated for an image reconstructed without DOI information, and a comparison with the resolution obtained with the full 4-layer DOI reconstruction is provided in Appendix A.1.

\subsubsection{Noise Equivalent Count Rate and Scatter Fraction The NECR and SF of} the scanner were evaluated using a cylindrical polyethylene phantom $(\varnothing=200 \mathrm{~mm}$, $h=700 \mathrm{~mm}$ ) with a line source inserted at a radial offset of $45 \mathrm{~mm}$. The phantom was positioned at the centre of the FOV, with its axis parallel to the axis of the scanner. List-mode data was collected for a range of activities (422.7 $\mathrm{MBq}$ to $1 \mathrm{MBq})$. Randoms were estimated using a delayed coincidence window method. The single slice rebinning algorithm was used to form prompt and delayed-coincidence sinograms. Count rates and SFs were evaluated from the sinograms and plotted as a function of effective activity concentration, defined in the NEMA NU 2-2012 standard as, the average source activity during the acquisition divided by the phantom volume.

2.3.4. Image Quality and Contrast Recovery A NEMA NU 2-2012 image quality phantom (IQP) was modelled in Geant4 and used for the evaluation of image quality and contrast recovery (CR) (DSC model PET/IEC-BODY/P with lung insert). The "warm" background volume of the phantom was filled with ${ }^{18} \mathrm{~F}$, with an activity concentration of $5.31 \mathrm{kBq} / \mathrm{mL}$. Four spherical "hot" lesions with diameters of $10 \mathrm{~mm}, 13 \mathrm{~mm}, 17 \mathrm{~mm}$ and $22 \mathrm{~mm}$ were filled with an activity concentration eight times greater than the background. Two spherical "cold" lesions with diameters of $28 \mathrm{~mm}$ and $37 \mathrm{~mm}$ were filled with non-radioactive water. A line source containing $72.1 \mathrm{MBq}$ of ${ }^{18} \mathrm{~F}$ was inserted into a cylindrical polyethylene phantom and positioned axially adjacent to the IQP to simulate background activity from outside of the FOV as shown in Figure 2.

Images were reconstructed using 3D ordinary-Poisson ordered-subset expectationmaximisation (OSEM) with 4 iterations and 8 subsets. Projection data was generated by forward projection using a system matrix in which the detector response was modelled using a simple Gaussian function [33]. The number of image voxels was $125 \times 125 \times 150$, with a voxel size of $3.0 \times 3.0 \times 3.0 \mathrm{~mm}^{3}$. A $6 \mathrm{~mm}$ FWHM Gaussian filter was applied to the reconstructed image. Random correction was performed using the delayed coincidence method. Attenuation correction factors were calculated directly based on knowledge of the phantom composition. Component-based normalisation was used, and single scatter simulation was applied to perform scatter correction.

The CR and background variability were also evaluated for an image reconstructed without DOI information, and a comparison with the results obtained using the full 4-layer DOI reconstruction is provided in Appendix A.2. 
A validated Geant4 model of a whole-body PET scanner with four-layer DOI detectors 12

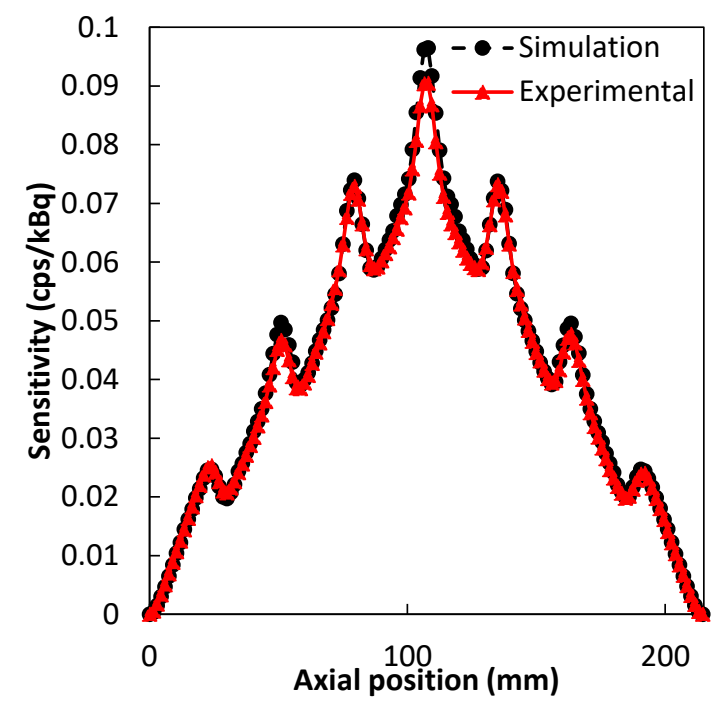

(a) Centre

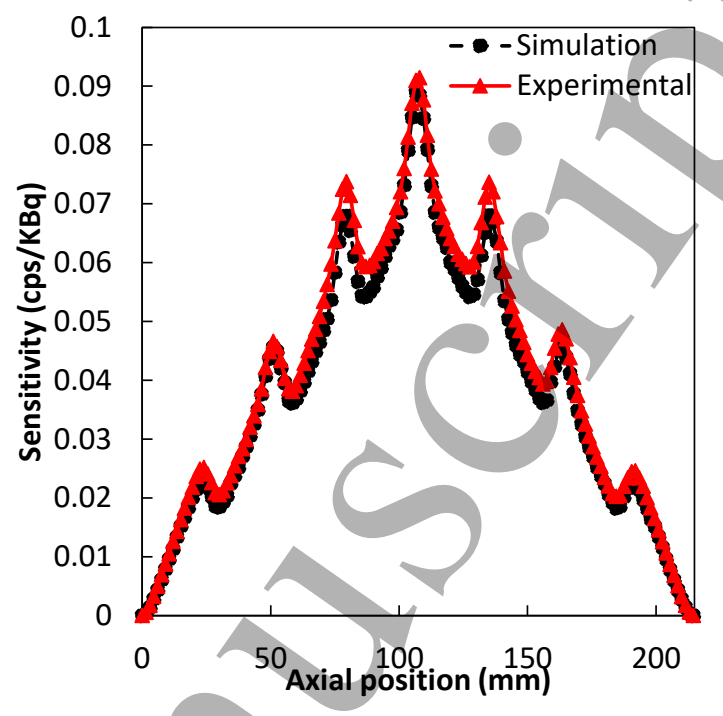

(b) Off-centre

Figure 6: Axial sensitivity profile of the line source in both evaluated positions.

\section{Results}

\subsection{Sensitivity}

The axial sensitivity profiles from the simulation are shown together with the corresponding experimental measurements (previously reported in [23]) in Figure 6. The system sensitivities for the experiment and simulation at the centre of the FOV were $5.9 \mathrm{cps} / \mathrm{kBq}$ and $6.2 \mathrm{cps} / \mathrm{kBq}$, respectively, and the sensitivities at $10 \mathrm{~cm}$ radial offset were $5.9 \mathrm{cps} / \mathrm{kBq}$ and $5.7 \mathrm{cps} / \mathrm{kBq}$ for the experiment and simulation, respectively. The physical scanner exhibits the same sensitivity at both locations, whereas sensitivity is slightly higher at the centre in the simulation.

\subsection{Spatial Resolution}

The values of the spatial resolution for the simulation are summarised in Table 2, together with the previously reported experimental values for the physical scanner. The average spatial resolutions of the simulation and experiment were $5.0 \pm 0.8 \mathrm{~mm}$ and $5.2 \pm 0.7 \mathrm{~mm}$, respectively.

\subsection{Noise Equivalent Count Rate and Scatter Fraction}

The count rate and the SF profiles of the simulated scanner are shown in Figure 7 and Figure 8, respectively, together with the experimental results from the physical scanner. The simulation and the experimental profiles are in close agreement except at high activity, where the counts significantly decrease in the case of experiment. The maximum NECR and SF for the experiment were $22.9 \mathrm{kcps}$ and $48.4 \%$, respectively, at 
A validated Geant4 model of a whole-body PET scanner with four-layer DOI detectors 13

Table 2: Spatial resolution for the simulated and physical scanners.

\begin{tabular}{lllll}
\hline Dimension & Offset $(\mathbf{m m})$ & Sim. & Phys. & Difference $(\%)$ \\
\hline \multirow{3}{*}{ Radial } & 10 & 4.0 & 4.1 & 2.8 \\
& 100 & 5.1 & 4.8 & -7.2 \\
& 200 & 6.4 & 5.9 & -8.8 \\
Tangential & 10 & 4.0 & 4.8 & +16.3 \\
& 100 & 4.1 & 4.7 & +13.7 \\
& 200 & 4.6 & 4.8 & +4.0 \\
Axial & 10 & 5.9 & 6.5 & +9.6 \\
& 100 & 5.1 & 5.7 & +9.7 \\
& 200 & 5.4 & 5.8 & +6.0 \\
\hline
\end{tabular}

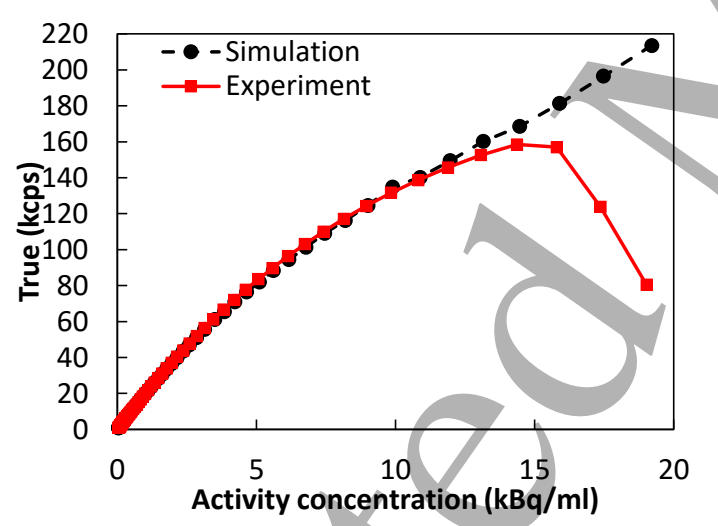

(a) True

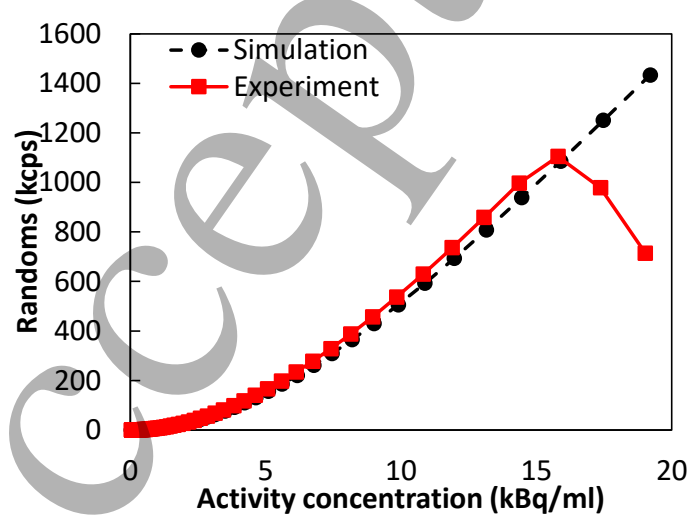

(c) Random

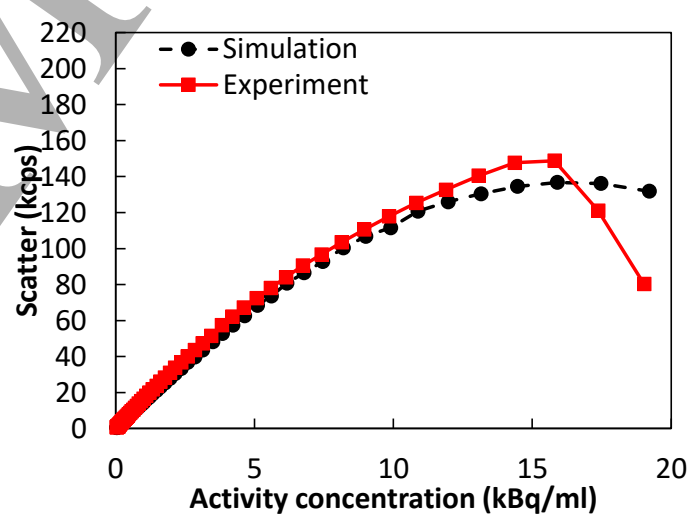

(b) Scatter

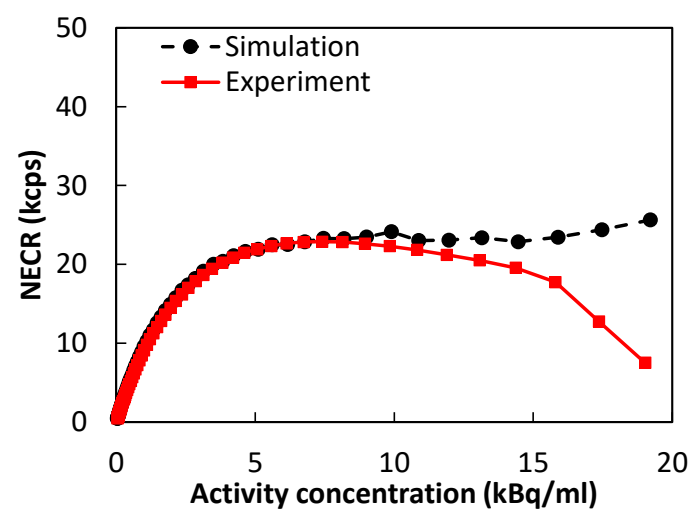

(d) NECR

Figure 7: Count rates of the whole-body PET scanner for the simulation and physical scanner. 
A validated Geant4 model of a whole-body PET scanner with four-layer DOI detectors 14

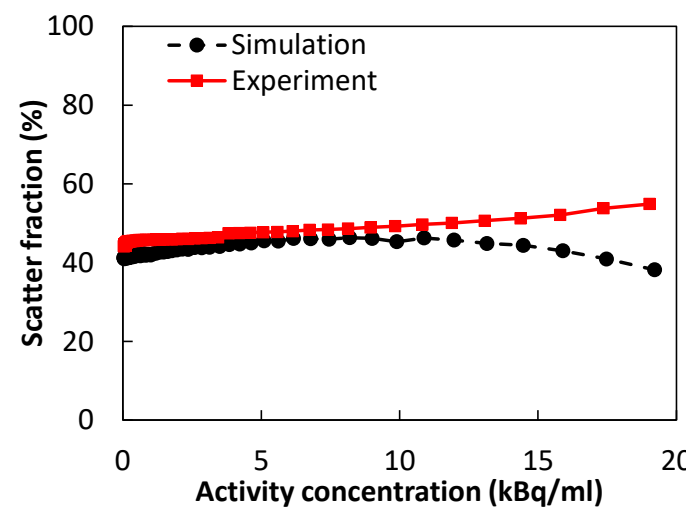

Figure 8: Scatter fraction profiles for the simulation and physical scanner.

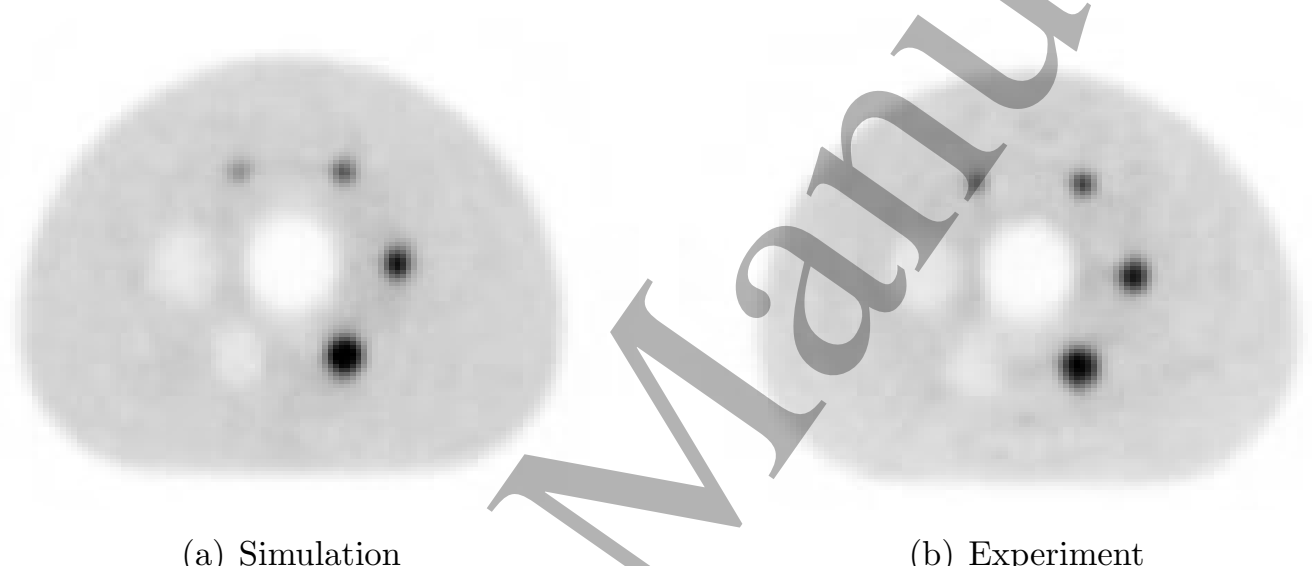

Figure 9: Reconstructed images of the image quality phantom.

332

an activity concentration of $7.45 \mathrm{kBq} / \mathrm{mL}$. The NECR curve for the simulation does not peak at a specific activity. The NECR and SF of the simulation at the peak activity of the experiment $(7.45 \mathrm{kBq} / \mathrm{mL})$ were $23.3 \mathrm{kcps}$ and $46.0 \%$, respectively. The SF of the experiment and simulation at low activity $(0.04 \mathrm{kBq} / \mathrm{mL})$ were $44.0 \%$ and $41.3 \%$, respectively.

\subsection{Image Quality and Contrast Recovery}

The reconstructed images of the IQP phantom are shown in Figure 9. The CR and background variability of the IQP for the simulated and physical scanners are listed in Table 3; the results are in close agreement. The average residual of the lung insert for a $30 \mathrm{~mm}$ region-of-interest (ROI) for the simulation was $5.4 \%$ compared to the experimental value of $9.1 \%$.

\section{4. Discussion}

The main objective of this work was to develop and validate a simulation model of the world's first 4-layer DOI-enabled whole-body prototype PET scanner developed at the 


\section{A validated Geant4 model of a whole-body PET scanner with four-layer DOI detectors 15}

Table 3: Comparison of contrast recovery and background variability.

\begin{tabular}{|c|c|c|c|c|}
\hline \multirow{2}{*}{$\begin{array}{c}\text { ROI } \\
\oslash(\mathrm{mm})\end{array}$} & \multicolumn{2}{|c|}{ Contrast (\%) } & \multicolumn{2}{|c|}{ Background variability (\%) } \\
\hline & Sim. & Phys. & Sim. & Phys. \\
\hline $10(\mathrm{~h})$ & 16.0 & 20.7 & 5.4 & 9.2 \\
\hline $13(\mathrm{~h})$ & 27.2 & 31.1 & 5.1 & 8.9 \\
\hline $17(\mathrm{~h})$ & 37.9 & 40.6 & 4.7 & 8.4 \\
\hline $22(\mathrm{~h})$ & 46.4 & 43.3 & 4.2 & 7.9 \\
\hline $28(\mathrm{c})$ & 34.2 & 33.2 & 3.9 & \\
\hline $37(\mathrm{c})$ & 41.4 & 39.9 & 3.6 & -0 \\
\hline
\end{tabular}

\section{NIRS-QST, Japan.}

The simulation demonstrated slightly higher sensitivity compared to the physical scanner at the centre of the FOV, as shown in Figure 6(a). The difference could be due to experimental error in the placement of the source. The absolute difference in sensitivity was $4.5 \%$ at the centre of the FOV and $4.1 \%$ at a radial offset of $10 \mathrm{~cm}$, indicating close agreement between the experiment and simulation.

The average magnitude of the error between the spatial resolutions obtained in the simulation and experiment was $5.1 \%$, which also shows a close agreement. The small error could be due to the assumption of a linear response shift of the PMT due to the reflector model being overly simplistic. Developing a more accurate look-up table based on a full optical photon simulation of a detector block will provide a more realistic model of the behaviour of this component. Finally, errors due to positioning of the point sources in the physical scanner during the experimental measurements could also contribute to the discrepancies in spatial resolution; however, it is noted that the discrepancy between the simulation and experimental spatial resolution is quite small compared to those previously reported in validation work on other PET scanners [11, 19].

All the count rate performances were in a close agreement except at the highest activities, where the physical scanner exhibits detector saturation as seen in Figure 7. The count rate errors between experiment and simulation were $0.8 \%$ for true, $4.1 \%$ for scatter, $5.9 \%$ for random and $1.8 \%$ for NECR at an activity concentration of $7.4 \mathrm{kBq} / \mathrm{mL}$ (where the peak NECR occurred for the physical scanner). A maximum NECR was not observed in the case of the simulation although a paralysable dead-time was applied on each block detector. This may be due to the idealised data scoring scheme used in the simulation in which the detectors continue accepting events without being dependent on factors such as limited bandwidth for data acquisition. In practice, the highest evaluated count rates do not occur in the application for which this scanner was designed (particle therapy quality assurance and related research), therefore the discrepancy in this region is not a major problem.

For the IQP (see Figure 9 and Table 3), the CR showed close agreement between the 


\section{A validated Geant4 model of a whole-body PET scanner with four-layer DOI detectors16}

simulated and physical scanners. The average error in CR for the hot regions was $-8.7 \%$ while for the cold regions it was $+3.4 \%$. The discrepancies observed in the background values are more substantial, with a consistent underestimation in the simulation $(-44 \%$ for hot lesions and $-48 \%$ for cold). This may be evidence of a background noise or error contribution (for example, noise in the experimental normalisation data or detector degradation) which is not currently included in the simulation and will be subject to future investigation.

The experimental NEMA NU 2 performance evaluation data used to validate the simulation model was acquired with the whole-body DOI PET scanner in the right cylindrical geometry (i.e. flat configuration) as shown in Figure 1(a). However, one of the unique characteristics of this scanner is that it can be mechanically reconfigured into an oblique-ring geometry to enable beam access for in-beam PET imaging. With the validation successfully completed, the simulation can now be extended to model the oblique-ring configuration as well, which will enable accurate simulationbased evaluation of clinical dose quantification and quality assurance algorithms to be performed.

To model readout bandwidth saturation at high activities, a saturation point (which, in this study, occurs at an activity concentration of $15.9 \mathrm{kBq} / \mathrm{mL}$ ) can be identified and a count loss model can be incorporated based on an empirical fit obtained from experimental results (since seyeral contributing factors in the data acquisition chain may account for the observed saturation). This will require more measurements to be performed on the real (physical) PET scanner and is the subject of ongoing experimental and simulation work.

\section{Conclusion}

A Geant4 model of a whole-body prototype PET scanner constructed from the four-layer DOI detector modules was validated against the experimental results obtained from the physical scanner, using a range of scanner performance metrics from the NEMA NU 2 2012 protocol. The sensitivities of the experiment and the simulation within the FOV showed an excellent agreement, with an average error of $4.3 \%$. The spatial resolutions of the simulation and the experiment were also in close agreement with average absolute error of $5.1 \%$. Count rate measurements closely matched the experimental values, except at very high activities, where the count-rate limitations of the physical scanner result in saturation. The peak NECR obtained with the physical scanner was $22.9 \mathrm{kcps}$ at an activity concentration of $7.4 \mathrm{kBq} / \mathrm{mL}$, while it was $23.3 \mathrm{kcps}$ for the simulation at the same activity concentration. The contrast recovery and background variability of the IQP also showed a close agreement with that of the experiment. Some of the differences between the experiment and simulation could be mitigated by incorporating more parameters of the prototype PET scanner into the Geant4 model. 


\section{A validated Geant4 model of a whole-body PET scanner with four-layer DOI detectors 17}

\section{Acknowledgements}

The authors would like to acknowledge the following organisations for providing access to their high performance computing resources: the Multi-modal/Australian Sciences Imaging and Visualisation Environment (MASSIVE) "M3" cluster and the Australian Nuclear Science and Technology Organisation (ANSTO) "Commodore" cluster. This research was undertaken with the assistance of resources from the National Computational Infrastructure (NCI Australia), an NCRIS-enabled capability supported by the Australian Government. The authors acknowledge the scientific and technical assistance of the National Imaging Facility, a National Collaborative Research Infrastructure Strategy (NCRIS) capability, at the Australian Nuclear Science and Technology Organisation, ANSTO.

\section{Appendix A. Effect of DOI Information on Imaging Performance}

\section{Appendix A.1. Spatial Resolution}

The spatial resolution of the scanner evaluated with and without incorporation of DOI information is given in Table A1 and Figure A1. The non-DOI results are obtained by collapsing the depths obtained in the DOI simulation to a single depth corresponding to the centroid of each $1 \times 4$ crystal column.

The radial spatial resolution of the simulation at $100 \mathrm{~mm}$ and $200 \mathrm{~mm}$ was degraded by $39.3 \%$ and $92.5 \%$, respectively, and that obtained in the experimental measurement was degraded at these depths by 44.2 and 94.4\%, respectively, as shown in Figure A1(a). This demonstrates the value of using DOI-sensitive detectors to improve the spatial resolution of the scanner at the periphery of the FOV. Tangential and axial spatial resolution are degraded by a much smaller amount by the loss of DOI information compared to radial spatial resolution.

\section{Appendix A.2. Image Quality}

The contrast recovery $(\mathrm{CR})$ coefficient obtained using the IQP with and without the use of DOI informiation is given Table A2 and Figure A2(a), and background variability is

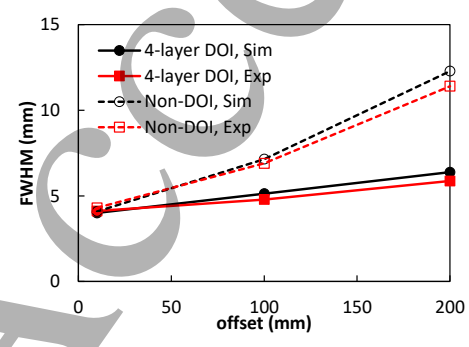

(a) Radial

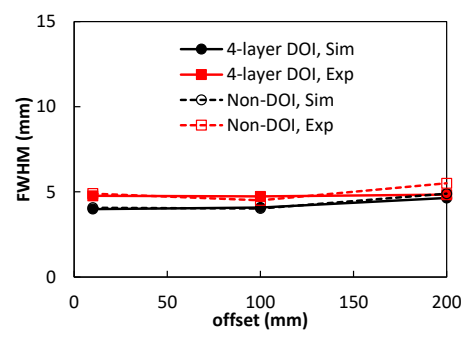

(b) Tangential

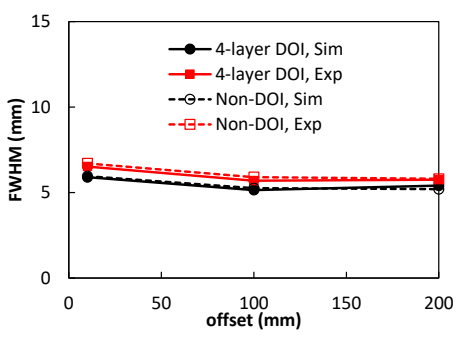

(c) Axial

Figure A1: Spatial resolution with and without DOI information. 
A validated Geant4 model of a whole-body PET scanner with four-layer DOI detectors18 Table A1: Spatial resolution obtained from images reconstructed with and without DOI information.

\begin{tabular}{|c|c|c|c|c|c|c|c|}
\hline \multirow{2}{*}{ Direction } & \multirow{2}{*}{$\begin{array}{l}\text { Offset } \\
(\mathrm{mm})\end{array}$} & \multicolumn{2}{|c|}{$\operatorname{Sim}(\%)$} & \multicolumn{2}{|l|}{ Phys. } & \multicolumn{2}{|c|}{$\begin{array}{l}\text { Difference } \\
(\%)\end{array}$} \\
\hline & & $\begin{array}{l}\text { 4-layer } \\
\text { DOI }\end{array}$ & $\begin{array}{l}\text { Non- } \\
\text { DOI }\end{array}$ & $\begin{array}{l}\text { 4-layer } \\
\text { DOI }\end{array}$ & $\begin{array}{l}\text { non- } \\
\text { DOI }\end{array}$ & $\begin{array}{l}\text { 4-layer } \\
\text { DOI }\end{array}$ & $\begin{array}{l}\text { Non- } \\
\text { DOI }\end{array}$ \\
\hline \multirow{3}{*}{ Radial } & 10 & 4.0 & 4.1 & 4.1 & & +2.8 & +5.0 \\
\hline & 100 & 5.1 & 7.1 & 4.8 & & -7.2 & -3.6 \\
\hline & 200 & 6.4 & 12.3 & 5.9 & & -8.8 & -7.8 \\
\hline \multirow{3}{*}{ Tangential } & 10 & 4.0 & 4.1 & 4.8 & & +16.3 & +16.9 \\
\hline & 100 & 4.1 & 4.0 & 4.7 & 5 & +13.7 & +10.6 \\
\hline & 200 & 4.6 & 4.9 & 4.8 & 5.5 & +4.0 & +11.1 \\
\hline \multirow{3}{*}{ Axial } & 10 & 5.9 & 6.0 & & & +9.6 & +11.1 \\
\hline & 100 & 5.1 & 5.3 & & 5.9 & +9.7 & +10.9 \\
\hline & 200 & 5.4 & 5.2 & & 5.8 & +6.0 & +10.4 \\
\hline
\end{tabular}

Table A2: Contrast recovery obtained with and without the use of DOI information.

\begin{tabular}{lllll}
\hline $\begin{array}{l}\text { ROI } \\
\oslash(\mathrm{mm})\end{array}$ & Sim. & & \multicolumn{3}{l}{ Phys. } \\
\cline { 2 - 3 } & $\begin{array}{l}\text { 4-layer } \\
\text { DOI }\end{array}$ & Non-DOI & $\begin{array}{l}\text { 4-layer } \\
\text { DOI }\end{array}$ & Non-DOI \\
\hline $10(\mathrm{~h})$ & 16.0 & 15.8 & 20.7 & 19.8 \\
$13(\mathrm{~h})$ & 27.2 & 26.7 & 31.1 & 30.6 \\
$17(\mathrm{~h})$ & 37.9 & 37.1 & 40.6 & 39.0 \\
$22(\mathrm{~h})$ & 46.4 & 45.4 & 43.3 & 42.0 \\
$28(\mathrm{c})$ & 34.2 & 34.6 & 33.2 & 37.1 \\
$37(\mathrm{c})$ & 41.4 & 41.7 & 39.9 & 42.3 \\
\hline
\end{tabular}

given in Table A3 and Figure A2(b). The CR and background variability values with and without DOI information only exhibit small differences. This is because the spheres used for this measurement are located at the centre of axial FOV. 
1

2

3

4

5

6

7

8

A validated Geant4 model of a whole-body PET scanner with four-layer DOI detectors 19

Table A3: Background variability obtained with and without the use of DOI information.

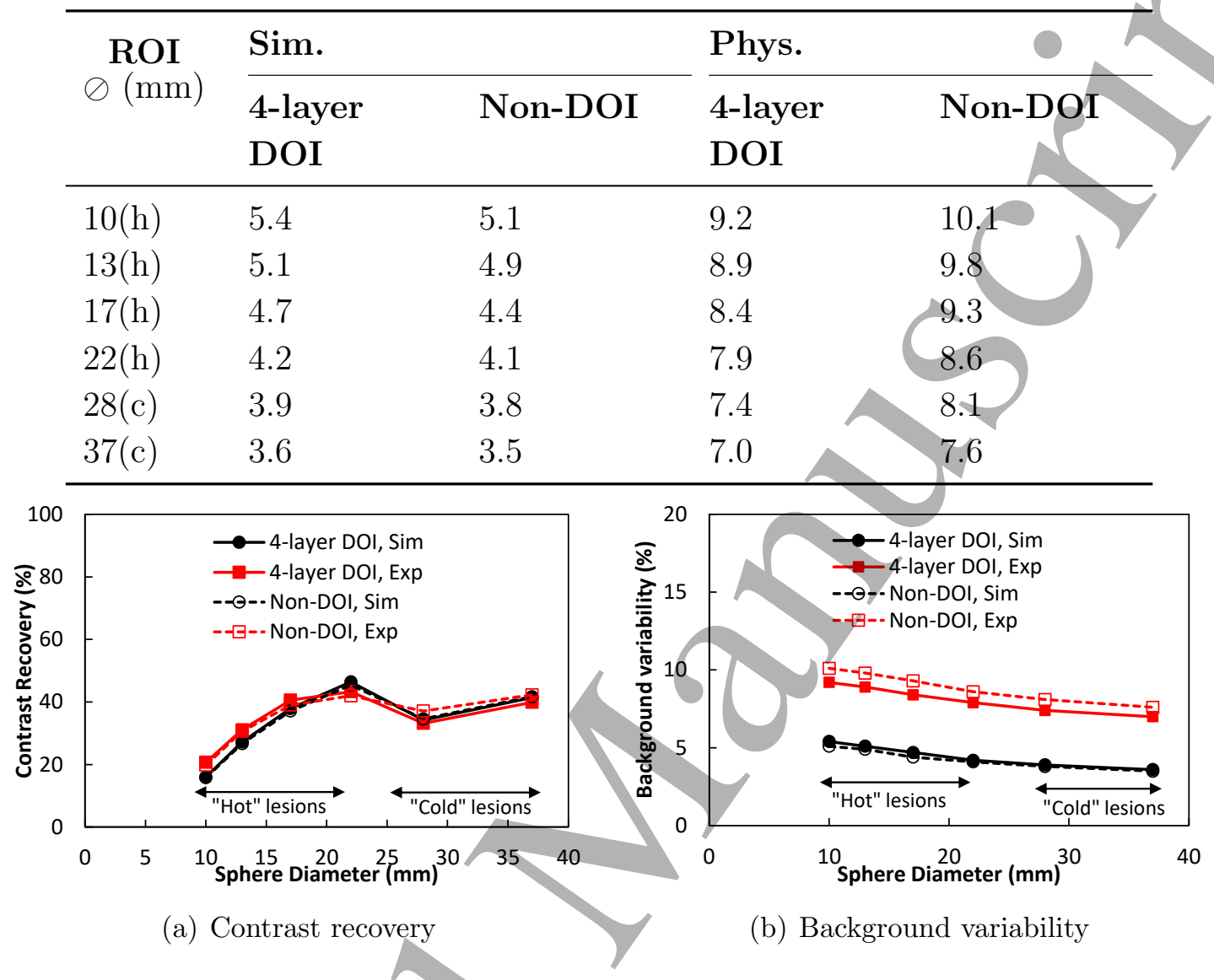

Figure A2: Contrast recovery and background variability for simulated and physical PET scanners, with and without the use of DOI effect.

\section{References}

[1] R. E. Schmitz, R. L. Harrison, C. W. Stearns, T. K. Lewellen, P. E. Kinahan, Optimization of noise equivalent count rate performance for a partially collimated pet scanner by varying the number of septa, IEEE Transactions on Medical Imaging 26 (2007) 935-944. doi:10.1109/TMI.2007.895485.

[2] S. Stute, T. Carlier, K. Cristina, C. Noblet, A. Martineau, B. Hutton, L. Barnden, I. Buvat, Monte carlo simulations of clinical pet and spect scans: impact of the input data on the simulated images, Physics in Medicine and Biology 56 (19) (2011) 6441-6457. doi:10.1088/00319155/56/19/017.

URL https://doi.org/10.1088/0031-9155/56/19/017

[3] L. Lu, H. Zhang, Z. Bian, J. Ma, Q. Feng, W. Chen, Validation of a Monte Carlo simulation of the Inveon PET scanner using GATE, Nuclear Instruments and Methods in Physics Research Section A: Accelerators, Spectrometers, Detectors and Associated Equipment 828 (2016) 170 175. doi:10.1016/j.nima.2016.04.059.

URL https://doi.org/10.1016/j.nima.2016.04.059

[4] J. E. Gillam, M. Rafecas, Monte-carlo simulations and image reconstruction for novel imaging scenarios in emission tomography, Nuclear Instruments and Methods in Physics Research Section A: Accelerators, Spectrometers, Detectors and Associated Equipment 809 (2016) 7688. doi:10.1016/j.nima.2015.09.084. 
A validated Geant4 model of a whole-body PET scanner with four-layer DOI detectors20

URL https://doi.org/10.1016/j.nima.2015.09.084

[5] E. Pfaehler, J. R. D. Jong, R. A. J. O. Dierckx, F. H. P. van Velden, R. Boellaard, SMART (SiMulAtion and ReconsTruction) PET: an efficient PET simulation-reconstruction tool, EJNMMI Physics 5 (1). doi:10.1186/s40658-018-0215-x. URL https://doi.org/10.1186/s40658-018-0215-x

[6] S. Jan, G. Santin, D. Strul, S. Staelens, K. Assié, D. Autret, S. Avner, R. Barbier, M. Bardiès, P. M. Bloomfield, D. Brasse, V. Breton, P. Bruyndonckx, I. Buvat, A. F. Chatziioannou, Y. Choi, Y. H. Chung, C. Comtat, D. Donnarieix, L. Ferrer, S. J. Glick, C. J. Groiselle, D. Guez, P.-F. Honore, S. Kerhoas-Cavata, A. S. Kirov, V. Kohli, M. Koole, M. Krieguer, D. J. van der Laan, F. Lamare, G. Largeron, C. Lartizien, D. Lazaro, M. C. Maas, L. Maigne, F. Mayet, F. Melot, C. Merheb, E. Pennacchio, J. Perez, U. Pietrzyk, F. R. Rannou, M. Rey, D. R. Schaart, C. R. Schmidtlein, L. Simon, T. Y. Song, J.-M. Vieira, D. Visvikis, R. V. de Walle, E. Wiers, C. Morel, GATE: a simulation toolkit for PET and SPECT, Physics in Medicine and Biology 49 (19) (2004) 4543-4561. doi:10.1088/0031-9155/49/19/007. URL https://doi.org/10.1088/0031-9155/49/19/007

[7] S. Jan, D. Benoit, E. Becheva, T. Carlier, F. Cassol, P. Descourt, T. Frisson, L. Grevillot, L. Guigues, L. Maigne, C. Morel, Y. Perrot, N. Rehfeld, D. Sarrut, D. R. Schaart, S. Stute, U. Pietrzyk, D. Visvikis, N. Zahra, I. Buvat, GATE v6: a major enhancement of the GATE simulation platform enabling modelling of $\mathrm{CT}$ and radiotherapy, Physics in Medicine and Biology 56 (4) (2011) 881-901. doi:10.1088/0031-9155/56/4/001. URL https : //doi.org/10.1088/0031-9155/56/4/001

[8] F. Lamare, A. Turzo, Y. Bizais, C. C. L. Rest, D. Visvikis, Validation of a monte carlo simulation of the philips allegro/GEMINI PET systems using GATE, Physics in Medicine and Biology 51 (4) (2006) 943-962. doi:10.1088/0031-9155/51/4/013. URL https://doi.org/10.1088/0031-9155/51/4/013

[9] R. L. Harrison, T. K. Lewellen, The simset program, in: Monte Carlo Calculations in Nuclear Medicine, Second Edition: Applications in Diagnostic Imaging, Taylor and Francis Group, 2013.

[10] R. L. Harrison, S. B. Gillispie, T. K. Lewellen, Design and implementation of a block detector simulation in SimSET, in: 2006 IEEE Nuclear Science Symposium Conference Record, IEEE, 2006, pp. 3151-3153. doi:10.1109/nssmic.2006.356543.

URL https://doi.org/10.1109/nssmic. 2006.356543

[11] J. K. Poon, M. L. Dahlbom, M. E. Casey, J. Qi, S. R. Cherry, R. D. Badawi, Validation of the SimSET simulation package for modeling the Siemens Biograph mCT PET scanner, Physics in Medicine and Biology 60 (3) (2015) N35-N45. doi:10.1088/0031-9155/60/3/n35. URL https : //doi.org/10.1088/0031-9155/60/3/n35

[12] M. Cañadas, P. Arce, P. R. Mendes, Validation of a small-animal PET simulation using GAMOS: a GEANT4-based framework, Physics in Medicine and Biology 56 (1) (2010) 273288. doi:10.1088/0031-9155/56/1/016. URL https://doi.org/10.1088/0031-9155/56/1/016

[13] P. Arce, J. I. Lagares, L. Harkness, D. Pérez-Astudillo, M. Cañadas, P. Rato, M. de Prado, Y. Abreu, G. de Lorenzo, M. Kolstein, A. Díaz, Gamos: A framework to do geant4 simulations in different physics fields with an user-friendly interface, Nuclear Instruments and Methods in Physics Research Section A: Accelerators, Spectrometers, Detectors and Associated Equipment 735 (2014) 304-313. doi:10.1016/j.nima.2013.09.036. URL https ://doi.org/10.1016/j.nima.2013.09.036

[14] S. España, J. L. Herraiz, E. Vicente, J. J. Vaquero, M. Desco, J. M. Udias, PeneloPET, a monte carlo PET simulation tool based on PENELOPE: features and validation, Physics in Medicine and Biology 54 (6) (2009) 1723-1742. doi:10.1088/0031-9155/54/6/021. URL https://doi .org/10.1088/0031-9155/54/6/021

[15] NEMA Standards Publication NU 2-1994: Performance measurements of positron emission tomographs, Washington DC, USA: National Electrical Manufacturers Association (NEMA), 
A validated Geant4 model of a whole-body PET scanner with four-layer DOI detectors21 2012.

[16] NEMA Standards Publication NU 2-2012: Performance measurements of positron emission tomographs, Rosslyn, USA: National Electrical Manufacturers Association (NEMA), 2012.

[17] NEMA Standards Publication NU 2-2018: Performance measurements of positron emission tomographs, Rosslyn USA: National Electrical Manufacturers Association (NEMA), 2018.

[18] P. Gonias, N. Bertsekas, N. Karakatsanis, G. Saatsakis, A. Gaitanis, D. Nikolopoulos, G. Loudos, L. Papaspyrou, N. Sakellios, X. Tsantilas, A. Daskalakis, P. Liaparinos, K. Nikita, A. Louizi, D. Cavouras, I. Kandarakis, G. Panayiotakis, Validation of a gate model for the simulation of the siemens biograph ${ }^{\mathrm{TM}} 6$ PET scanner, Nuclear Instruments and Methods in Physics Research Section A: Accelerators, Spectrometers, Detectors and Associated Equipment 571 (1-2) (2007) 263-266. doi:10.1016/j.nima.2006.10.078. URL https://doi.org/10.1016/j.nima.2006.10.078

[19] A. Ghabrial, D. Franklin, H. Zaidi, A monte carlo simulation study of the impact of novel scintillation crystals on performance characteristics of pet scanners, Physica Medica 50 (2018) 37-45. doi:10.1016/j.ejmp.2018.05.010. URL https://doi.org/10.1016/j.ejmp.2018.05.010

[20] T. Tsuda, H. Murayama, K. Kitamura, T. Yamaya, E. Yoshida, T. Omura, H. Kawai, N. Inadama, N. Orita, A four-layer depth of interaction detector block for small animal pet, IEEE Transactions on Nuclear Science 51 (5) (2004) 2537-2542. doi:10.1109/tns.2004.835739. URL https://doi.org/10.1109/tns.2004.835739

[21] Y. Hirano, M. Nitta, N. Inadama, F. Nishikido, E. Yoshida, H. Murayama, T. Yamaya, Performance evaluation of a depth-of-interaction detector by use of position-sensitive PMT with a super-bialkali photocathode, Radiological Physics and Technology 7 (1) (2013) 57-66. doi:10.1007/s12194-013-0231-4. URL https ://doi .org/10.1007/s12194-013-0231-4

[22] H. Tashima, T. Yamaya, E. Yoshida, S. Kinouchi, M. Watanabe, E. Tanaka, A single-ring OpenPET enabling PET imaging during radiotherapy, Physics in Medicine and Biology 57 (14) (2012) 4705-4718. doi:10.1088/0031-9155/57/14/4705. URL https : //doi.org/10.1088/0031-9155/57/14/4705

[23] G. Akamatsu, H. Tashima, Y. Iwao, H. Wakizaka, T. Maeda, A. Mohammadi, S. Takyu, M. Nitta, F. Nishikido, H. Rutherford, A. Chacon, M. Safavi-Naeini, E. Yoshida, T. Yamaya, Performance evaluation of a whole-body prototype PET scanner with four-layer DOI detectors, Physics in Medicine and Biologydoi:10.1088/1361-6560/ab18b2. URL https ://doi.org/10.1088/1361-6560/ab18b2

[24] T. Hofmann, M. Pinto, A. Mohammadi, M. Nitta, F. Nishikido, Y. Iwao, H. Tashima, E. Yoshida, A. Chacon, M. Safavi-Naeini, A. Rosenfeld, T. Yamaya, K. Parodi, Dose reconstruction from PET images in carbon ion therapy: a deconvolution approach, Physics in Medicine \& Biology 64 (2) (2019) 025011. doi:10.1088/1361-6560/aaf676. URL https://doi.org/10.1088/1361-6560/aaf676

[25] T. Hofmann, A. Fochi, K. Parodi, M. Pinto, Prediction of positron emitter distributions for range monitoring in carbon ion therapy: an analytical approach, Physics in Medicine \& Biologydoi:10.1088/1361-6560/ab17f9. URL https://doi .org/10.1088/1361-6560/ab17f9

[26] A.Chacon, S. Guatelli, H. Rutherford, D. Bolst, A. Mohammadi, A. Ahmed, M. Nitta, F. Nishikido, Y. Iwao, H. Tashima, E. Yoshida, G. Akamatsu, S. Takyu, A. Kitagawa, T. Hofmann, M. Pinto, D. R. Franklin, K. Parodi, T. Yamaya, A. Rosenfeld, M. Safavi-Naeini, Comparative study of alternative geant4 hadronic ion inelastic physics models for prediction of positron-emitting radionuclide production in carbon and oxygen ion therapy, Physics in Medicine \& Biology 64 (15) (2019) 155014. doi:10.1088/1361-6560/ab2752. URL https : //doi .org/10.1088/1361-6560/ab2752

[27] A. Chacon, M. Safavi-Naeini, D. Bolst, S. Guatelli, D. R. Franklin, Y. Iwao, G. Akamatsu, 


\section{A validated Geant4 model of a whole-body PET scanner with four-layer DOI detectors22}

H. Tashima, E. Yoshida, F. Nishikido, et al., Monte carlo investigation of the characteristics of radioactive beams for heavy ion therapy, Scientific reports 9 (1) (2019) 6537.

[28] A. Chacon, B. James, L. Tran, S. Guatelli, L. Chartier, D. Prokopovich, D. Franklin, A. Mohammadi, F. Nishikido, Y. Iwao, G. Akamatsu, S. Takyu, H. Tashima, T. Yamaya, K. Parodi, A. Rosenfeld, M. Safavi-Naeini, Experimental investigation of the characteristics of radioactive beams for heavy ion therapy, Medical Physics.

[29] T. Yamaya, H. Tashima, OpenPET enabling PET imaging during radiotherapy, in: Personalized Pathway-Activated Systems Imaging in Oncology, Springer Singapore, 2017, pp. 55-84. doi:10.1007/978-981-10-3349-0_4. URL https://doi .org/10.1007/978-981-10-3349-0\_4

[30] S. Agostinelli, J. Allison, K. Amako, J. Apostolakis, et al., Geant4-a simulation toolkit, Nuclear Instruments and Methods in Physics Research Section A: Accelerators, Spectrometers, Detectors and Associated Equipment 506 (3) (2003) 250-303. doi:10.1016/s0168-9002(03)01368-8. URL https://doi.org/10.1016/s0168-9002(03)01368-8

[31] J. Allison, K. Amako, J. Apostolakis, et al., Recent developments in Geant4, Nuclear Instruments and Methods in Physics Research Section A: Accelerators, Spectrometers, Detectors and Associated Equipment 835 (2016) 186-225. doi:10.1016/j.nima.2016.06.125.

URL https://doi.org/10.1016/j.nima.2016.06.125

[32] N. Shimura, M. Kamada, A. Gunji, S. Yamana, T. Usui, K. Kurashige, H. Ishibashi, N. Senguttuvan, S. Shimizu, K. Sumiya, H. Murayama, Zr doped GSO:ce single crystals and their scintillation performance, IEEE Transactions on Nuclear Science 53 (5) (2006) 2519-2522. doi:10.1109/tns.2006.876006. URL https://doi.org/10.1109/tns.2006.876006

[33] S. Kinouchi, T. Yamaya, E. Yoshida, H. Tashima, H. Kudo, H. Haneishi, M. Suga, Gpu-based pet image reconstruction using an accurate geometrical system model, IEEE Transactions on Nuclear Science 59 (5) (2012) 1977-1983. doi:10.1109/tns.2012.2201953.

URL https://doi.org/10.1109/tns.2012,2201953 